\title{
Impact of fracture clustering on the seismic signatures of porous rocks containing aligned fractures
}

\author{
Nicolás D. Barbosa ${ }^{1}$, J. Germán Rubino ${ }^{2}$, Eva Caspari ${ }^{1}$, and Klaus Holliger ${ }^{1}$
}

(March 23, 2021)

Running head: Fracture clustering effects on WIFF

\begin{abstract}
The presence of fractures in a reservoir can have a significant impact on its effective mechanical and hydraulic properties. Many researchers have explored the seismic response of fluid-saturated porous rocks containing aligned planar fractures through the use of analytical models. However, these approaches are limited to the extreme cases of regular and uniform random distributions of fractures. The purpose of this work is to consider more realistic distributions of fractures and to analyze whether and how the frequency-dependent anisotropic seismic properties of the medium can provide information on the characteristics of the fracture network. Particular focus is given to fracture clustering effects resulting from commonly observed fracture distributions. To do so, we propose a novel hybrid methodology combining the advantages of 1D numerical oscillatory tests, which allows us to consider arbitrary distributions of fractures, and an analytical solution, which permits to extend these results to account for the effective anisotropy of the medium. A corresponding numerical

\footnotetext{
${ }^{1}$ Applied and Environmental Geophysics Group, Institute of Earth Sciences, University of Lausanne, Lausanne, Switzerland

${ }^{2}$ CONICET, Centro Atómico Bariloche - CNEA, San Carlos de Bariloche, Argentina

${ }^{3}$ Accepted for publication in Geophysics.
} 
analysis shows that the presence of clusters of fractures produces an additional attenuation and velocity dispersion regime compared to that predicted by analytical models. The reason for this is that a fracture cluster behaves as an effective layer and the contrast with respect to the unfractured background produces an additional fluid pressure diffusion length scale. The characteristic frequency of these effects depends on the size and spacing between clusters, the latter being much larger than the typical spacing between individual fractures. Moreover, we find that the effects of fracture clustering are more pronounced in attenuation anisotropy than velocity anisotropy data. Our results show that fracture clustering effects on fluid pressure diffusion can be described by two-layer models. This, in turn, provides the basis for extending current analytical models to account for these effects in inversion schemes designed to characterize fractured reservoirs from seismic data. 


\section{INTRODUCTION}

Most reservoirs are assumed to contain a set of sub-vertical fractures (Liu and Martinez, 2013) because open fractures tend to be oriented normal to the direction of minimum in-situ compressive stress (Schoenberg and Sayers, 1995), which in the considered depth range is usually quasi-horizontal. Thus, understanding the impact of the presence of subvertical fractures on the seismic properties of a reservoir is a topic of great interest in the field of Exploration Geophysics. Probably the most remarkable seismic manifestation of the presence of a set of aligned vertical fractures in an otherwise isotropic background rock is the effective transverse isotropy with a horizontal axis of symmetry (HTI). Bakulin et al. (2000) provide a detailed review of the two classical approaches to the study of such media, that is, elastic effective models based on parallel infinite fractures represented as linear slip boundary conditions (Schoenberg, 1980) and penny-shaped crack models (e.g., Hudson, 1980; Thomsen, 1995). Based on these models, it is possible to retrieve some key parameters of fractured formations from seismic recordings such as, for example, fracture density, azimuthal fracture orientation, and type of saturating pore fluid. In all these works, the effective medium is represented by an elastic solid, which in turn implies that the effective stiffness tensor of the medium is frequency-independent and real-valued.

However, the presence of fluids in the pore space of rocks causes seismic attenuation and velocity dispersion due to a mechanism broadly known as wave-induced fluid flow (WIFF). In particular, mesoscopic WIFF, which occurs due to spatial variations in rock compliance on a scale much larger than the pore size but much smaller than the wavelength, is considered to be a significant source of seismic energy dissipation and velocity dispersion in the seismic frequency band (Müller et al., 2010; Krzikalla and Müller, 2011). In rocks contain- 
ing mesoscopic fractures, the large mechanical contrast between compliant fractures and the stiff background favours the development of particularly strong fluid pressure gradients between these regions in response to the propagation of a seismic wave. Consequently, fluid flows accompanied with internal friction until fluid pressure equilibrates. This phenomenon results in frequency-dependent and complex-valued effective stiffness components representing the anisotropic medium (e.g., Rubino et al., 2016).

White et al. (1975) studied mesoscopic WIFF effects on the propagation of normally incident P-waves in fluid-saturated periodically layered porous media. Brajanovski et al. (2005) used the results from White et al. (1975) to explore the case of a regular distribution of parallel fractures, which are represented as a limiting case of very thin and highly porous layers in a less porous background. Furthermore, this model assumes that fractures have infinite extent and that the spacing between fractures is much larger than the fracture aperture. The latter represents a strong limitation of these types of models as they are strictly valid only for low fracture densities. Using numerical simulations, Lambert et al. (2006) demonstrated the validity of this analytical model for periodic distributions of fractures with constant aperture. Recently, Kong et al. (2013) and Guo et al. (2017) generalized the model of Brajanovski et al. (2005) to account for different fluids saturating the fractures and the background and to the case of finite fracture thickness, respectively.

In the case of thinly layered porous media, Krzikalla and Müller (2011) showed that the full effective stiffness tensor can be computed from the effective P-wave modulus normal to the fractures. This is due to the fact that, as the slow $\mathrm{P}$-wave velocity is much lower than that of classic elastic waves, the fluid pressure diffusion between the thin layers and the background is mainly normal to the surface of the layers for any direction of propagation of the incident wave. Hence, the frequency dependence of the five independent complex- 
valued stiffness elements can be represented by a single relaxation function connecting their relaxed and unrelaxed regimes. Several authors have used this idea to study the frequencydependent anisotropy of transversely isotropic media. For example, Carcione et al. (2013) used the effective P-wave moduli given by the models of White et al. (1975) and Brajanovski et al. (2005), whereas Galvin and Gurevich (2015) used the penny-shaped crack model of Gurevich et al. (2009). However, these works are based on analytical solutions which have limitations with regard to the distribution, thickness and/or density of fractures or assume extremely high contrasts of the elastic and hydraulic properties between the fractures and the background. A methodology that has proven to be useful to overcome these issues to compute the effective properties of arbitrarily heterogeneous porous rocks is numerical upscaling (Masson and Pride, 2007; Rubino et al., 2009; Wenzlau et al., 2010).

In real reservoirs, fractures often exhibit complex distributions and, hence, quantitative characterizations of realistic spatial distributions of fractures are needed for a correct interpretation of seismic measurements. Several authors have measured and studied fracture spacing distributions, ranging from non-fractal to fractal, from 1D fracture distributions (e.g., Huang and Angelier, 1989; Gillespie et al., 1993). Fang et al. (2016) showed that in the presence of irregularly distributed fractures, clusters of fractures can occur, that is, groups of closely spaced fractures separated by relatively unfractured rock. Although this is a rather common scenario in fractured rocks (Liu and Martinez, 2013), the analysis of the impact of fracture clustering on the WIFF effects with regard to seismic wave anisotropy remains largely unexplored. This is likely to be a consequence of the limitations of analytical models, on the one hand, and the high computational cost of the currently available numerical methodologies for complex fracture distributions, on the other hand.

In this work, we analyze the seismic properties of fluid-saturated rocks containing ran- 
dom distributions of aligned planar fractures, with particular emphasis on fracture clustering effects. To do so, we propose a novel hybrid methodology to determine the effective anisotropic behavior of the elastic wave modes propagating through a fluid-saturated porous rock containing an arbitrary distribution of aligned fractures. We compute the effective Pwave modulus normal to the fractures through the application of a numerical upscaling procedure based on a 1D oscillatory relaxation test. Once the effective $\mathrm{P}$-wave modulus is obtained, we make use of the uni-dimensional nature of fluid pressure diffusion processes in layered media (Krzikalla and Müller, 2011) to obtain the full stiffness tensor of the effective TI medium representing the fractured rock. The stiffness tensor allows us to study the frequency-dependent anisotropic seismic signatures of the probed fractured reservoir. By doing so, we significantly reduce the computational cost with respect to performing several 3D numerical relaxation tests such as those presented by Wenzlau et al. (2010). For the analysis of fracture clustering effects on the effective seismic properties of the medium, we estimate the effective physical properties as well as the apertures of the considered planar fractures from the ultrasonic velocities measured in a synthetic sample containing pennyshaped cracks by Rathore et al. (1995). We start the analysis of fracture clustering effects with the effective $\mathrm{P}$-wave modulus at normal incidence to understand the general characteristics of the fluid pressure relaxation process. Then, we continue by analyzing the effects on the effective seismic anisotropy of the medium.

\section{METHODOLOGY}

In this section, we present the methodology to determine the complex-valued frequencydependent effective stiffness tensor of poroelastic rocks containing aligned planar fractures. It has been shown that when an otherwise isotropic rock contains a distribution of fluid- 
saturated fractures that have preferred orientations, the resulting material will exhibit an effective frequency-dependent seismic anisotropy (Liu and Martinez, 2013). Indeed, the effective seismic response of such a medium is transversely isotropic. To compute the phase velocity and inverse quality factor as functions of frequency and direction of wave propagation, it is necessary to determine the effective stiffness tensor of the medium. To do so, we propose a hybrid approach, which consists of (i) computing the effective frequencydependent $\mathrm{P}$-wave modulus in the direction normal to the fractures through the application of a 1D numerical upscaling procedure (Figure 1) and (ii) analytically obtaining all the frequency-dependent stiffness elements from their corresponding low- and high-frequency limits and the relaxation behavior of the effective P-wave modulus at normal-to-fracture incidence (Krzikalla and Müller, 2011).

In analogy to previous works on fluid-saturated fractured media (e.g., Brajanovski et al., 2005; Gurevich et al., 2009; Carcione et al., 2013; Rubino et al., 2013), we model the response of fractured rocks in the framework of Biot's (1962) theory of poroelasticity by representing the fractures as highly compliant and permeable thin layers embedded in a stiffer and less permeable matrix. As planar fractures are assumed to have infinite lateral extension, this fracture model is strictly valid when the fractures radii are much larger than the prevailing wavelengths and the spacing between consecutive fractures (Gurevich et al., 2009).

\section{Effective $\mathrm{P}$-wave modulus at normal-to-fracture incidence}

The effective $\mathrm{P}$-wave modulus in the direction normal to the fractures is obtained by applying time-harmonic solid displacements to the boundaries of a representative elementary volume (REV) of the fractured medium of interest (Figure 1). The resulting stress and 
strain fields in the sample are computed based on a numerical solution of the quasi-static approximation of Biot's (1962) isotropic equations. The reasoning behind this is that, for frequencies much smaller than Biot's critical frequency, the physical process is controlled by fluid-pressure diffusion and thus inertial effects can be neglected. Hence, the stress equilibrium and Darcy's law are to be fulfilled simultaneously. Moreover, for the considered case of a deformation applied normally to a set of planar fractures, plane-strain conditions are satisfied. Hence, in the space-frequency domain and assuming that the fractures are normal to the $x$-axis, the problem reduces to solving the $1 \mathrm{D}$ equations

$$
\begin{aligned}
\frac{d \tau_{x x}}{d x} & =0 \\
-\frac{d p_{f}}{d x} & =i \omega \frac{\eta}{\kappa} w_{x}
\end{aligned}
$$

where $\tau_{x x}, w_{x}$, and $p_{f}$ are the $1 \mathrm{D}$ total stress, the average relative fluid displacement and the fluid pressure, respectively, $\eta$ is the fluid viscosity, $\kappa$ the permeability and $\omega$ the angular frequency. These equations are coupled through the 1D constitutive relations for $\tau_{x x}$ and $p_{f}$, which for an isotropic porous medium are given by

$$
\begin{gathered}
\tau_{x x}=(2 \mu+\lambda) \varepsilon_{x x}-\alpha M \zeta, \\
p_{f}=-\alpha M \varepsilon_{x x}+M \zeta,
\end{gathered}
$$

where $u_{x}$ and $\varepsilon_{x x}=\frac{d u_{x}}{d x}$ are the $1 \mathrm{D}$ displacement and strain of the solid phase, respectively, and $\zeta=-\frac{d w_{x}}{d x}$ is a measure of local change in the fluid content. The material parameters as well as the displacements $u_{x}$ and $w_{x}$ are functions of a single coordinate $x$. In Equation $2, \mu$ is the shear modulus of the saturated material, which is equal to that of the dry frame $\mu_{m}$. The so-called Biot-Willis effective stress coefficient $\alpha$, the Biot's fluid-storage modulus 
$M$, and the undrained Lamé constant $\lambda$ are defined as

$$
\begin{aligned}
\alpha & =1-K_{m} / K_{s}, \\
M & =\left(\frac{\alpha-\phi}{K_{s}}+\frac{\phi}{K_{f}}\right)^{-1}, \\
\lambda & =K_{m}-\frac{2}{3} \mu+\alpha^{2} M,
\end{aligned}
$$

with $\phi$ being the porosity and $K_{f}, K_{s}$, and $K_{m}$ the bulk moduli of the fluid phase, the solid grains, and the dry matrix, respectively.

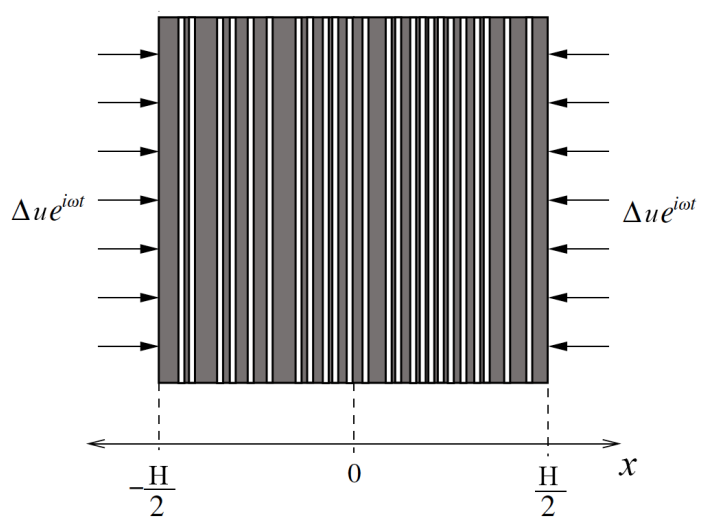

Figure 1: Schematic illustration of the oscillatory relaxation test employed for determining the effective P-wave modulus in the direction normal to the fractures. Grey and white regions represent background and fractures, respectively.

Let $\Omega=\left(-\frac{H}{2}, \frac{H}{2}\right)$ be a domain that represents the probed sample. We thus impose a homogeneous time-harmonic solid displacement at the hydraulically sealed boundaries of the sample, that is,

$$
\begin{aligned}
& u_{x}=\Delta u, \quad \text { at } x=-\frac{H}{2}, \\
& u_{x}=-\Delta u, \quad \text { at } x=\frac{H}{2}, \\
& w_{x}=0, \quad \text { at } \quad x=-\frac{H}{2}, \frac{H}{2} .
\end{aligned}
$$


By employing a finite-element procedure to solve Equations 1 and 2 under the imposed boundary conditions (Equation 4), we obtain the solid and relative fluid displacement fields in the sample as functions of frequency. From these fields, we compute the stress field using Equation 2 and the strain field as the spatial derivative of the solid displacement. Under the dynamic-equivalent viscoelastic medium assumption (e.g. Solazzi et al., 2016), the effective $\mathrm{P}$-wave modulus in the direction normal to the fractures is thus obtained from

$$
C_{11}(\omega)=\frac{\left\langle\tau_{x x}(\omega)\right\rangle}{\left\langle\epsilon_{x x}(\omega)\right\rangle}
$$

where the spatial averages of the local stress and strain fields over the total length $H$ of the sample are

$$
\begin{aligned}
& \left\langle\tau_{x x}(\omega)\right\rangle=\frac{1}{H} \int_{\Omega} \tau_{x x}\left(\omega, x^{\prime}\right) \mathrm{d} x^{\prime}, \\
& \left\langle\epsilon_{x x}(\omega)\right\rangle=\frac{1}{H} \int_{\Omega} \epsilon_{x x}\left(\omega, x^{\prime}\right) \mathrm{d} x^{\prime}=\frac{-2 \Delta u}{H},
\end{aligned}
$$

respectively. The effective modulus computed in Equation 5 quantifies the response of the fractured porous rock of interest to a $\mathrm{P}$-wave propagating normal to the fractures. It is important to remark that the concept of an effective medium assumes that the prevailing wavelengths are much larger than the correlation length of the considered random distribution of fractures.

\section{Effective stiffness tensor}

Since most reservoirs are assumed to contain a set of sub-vertical fractures (Maultzsch et al., 2003; Liu and Martinez, 2013; Gale et al., 2014), we study the case of a horizontal symmetry axis and, thus, the medium has effective horizontal transverse isotropy. Following Figure 1, the symmetry axis, normal to the fracture planes, is parallel to the $x$-axis and, hence, the 
effective constitutive relations can be written as

$$
\left[\begin{array}{c}
\tau_{x x} \\
\tau_{y y} \\
\tau_{z z} \\
\tau_{y z} \\
\tau_{x z} \\
\tau_{x y}
\end{array}\right]=\left[\begin{array}{cccccc}
C_{11} & C_{12} & C_{12} & 0 & 0 & 0 \\
C_{12} & C_{33} & C_{23} & 0 & 0 & 0 \\
C_{12} & C_{23} & C_{33} & 0 & 0 & 0 \\
0 & 0 & 0 & \left(C_{33}-C_{23}\right) / 2 & 0 & 0 \\
0 & 0 & 0 & 0 & C_{66} & 0 \\
0 & 0 & 0 & 0 & 0 & C_{66}
\end{array}\right] \cdot\left[\begin{array}{c}
\varepsilon_{x x} \\
\varepsilon_{y y} \\
\varepsilon_{z z} \\
2 \varepsilon_{y z} \\
2 \varepsilon_{x z} \\
2 \varepsilon_{x y}
\end{array}\right],
$$

where the $C_{i j}$ stiffness coefficients can be complex-valued and frequency-dependent. Using that, in this kind of scenario, the fluid pressure diffusion is approximately normal to the surface of the fractures, Krzikalla and Müller (2011) found that the frequency dependence of the $C_{i j}$ coefficients is given by a single scalar relaxation function $R(\omega)$, such that the effective stiffness tensor has the form

$$
C_{i j}(\omega)=C_{i j}^{u}-R(\omega) \cdot\left[C_{i j}^{u}-C_{i j}^{r}\right]
$$

That is, the anisotropy of the effective stiffness is determined by the unrelaxed and relaxed limits of the stiffness coefficients, $C_{i j}^{u}$ and $C_{i j}^{r}$, respectively, and a complex-valued function $R(\omega)$ describing the transition between both regimes. Gelinsky and Shapiro (1997) obtained the effective stiffness tensor for layered media for the unrelaxed and relaxed limits in a Backus-type formulation. In Appendix A, we provide the expressions for the relaxed and unrelaxed limits of the stiffness elements for this kind of medium. In order to obtain the relaxation function of an arbitrary distribution of aligned fractures, we use Equation 8, the numerically computed effective P-wave modulus of the medium $C_{11}(\omega)$ (Equation 5), and its low- and high-frequency limits as follows

$$
R(\omega)=\frac{C_{11}(\omega)-C_{11}^{u}}{C_{11}^{r}-C_{11}^{u}}
$$


Using Equations 8, 9, and Appendix A, the $C_{i j}(\omega)$ coefficients representing the medium of interest are determined. We then compute the phase velocity and seismic attenuation of the compressional and shear waves as functions of frequency and direction of wave propagation (Appendix B).

Krzikalla and Müller (2011) demonstrated the uni-dimensionality of the fluid pressure diffusion between the background and aligned layers, and hence, the validity of Equation 8 considering two- and three-layer models. To do so, they computed the relaxation functions of the effective stiffness elements following the numerical methodology presented in Wenzlau et al. (2010). By comparing the relaxation functions, they found that they were the same for all the stiffness elements, which confirms the validity of Equation 8. Given that our methodology allows to consider more complex scenarios, we further generalize the validity of Equation 8 for the case of a medium containing clusters of fractures (Appendix C). In that case, four characteristic lengths are present, namely the fracture thickness, the separation between adjacent fractures, the effective thickness of the cluster, and the distance between consecutive clusters of fractures. The validation is carried out by comparison with the effective stiffness tensor elements obtained from 3D relaxation tests.

It is important to remark that, the main advantage of this hybrid approach with respect to available analytical models is that it allows to handle arbitrary fracture sets without the limitations commonly associated with analytical solutions, such as, for example, low fracture density. Furthermore, it is valid for generic horizontally stratified poroelastic systems which, in turn, allows to consider hydraulically and elastically heterogeneous backgrounds as well as arbitrary property contrasts between the fractures and the background. 


\section{FRACTURE PHYSICAL PROPERTIES IN A POROELASTIC CONTEXT}

As mentioned before, we conceptualize fractures as very thin fluid-saturated porous layers. Given that the fluid pressure diffusion process between the fractures and the background strongly depends on their mechanical contrast, the elastic properties of the fractures' dry frame are key parameters for the numerical analysis. For simplicity, many authors (e.g., Rubino et al., 2013; Quintal et al., 2014; Guo et al., 2017) have used the values published by Nakagawa and Schoenberg (2007) for the shear and dry normal fracture compliances as a reference to determine the fractures' elastic properties. In this work, we chose fracture and background properties based on velocities experimentally measured by Rathore et al. (1995) for a synthetic sandstone containing aligned penny-shaped cracks. Given that a model of aligned infinite fractures and a model of aligned penny-shaped cracks yield the same structure for the effective stiffness tensor (Wenzlau et al., 2010), we can define medium parameters to obtain identical phase velocities under dry conditions for both models (Schoenberg and Douma, 1988). However, the frequency dependence of attenuation and velocity under fluid-saturated conditions will be different for a penny-shaped crack model and a planar fracture model. This is due to the fact that for planar fractures the pattern of conversion to diffusive waves is $1 \mathrm{D}$, whereas finite fractures act essentially as $3 \mathrm{D}$ features and, hence, fluid pressure can diffuse in all directions (Wenzlau et al., 2010).

The samples used by Rathore et al. (1995) were manufactured by embedding thin metal discs into a sand-epoxy matrix, which were chemically leached out later on, thus leaving penny-shaped voids. These voids have thicknesses of $0.02 \mathrm{~mm}$ and are aligned and regularly

spaced every $2 \mathrm{~mm}$. P- and S-wave velocities were measured as a function of angle from 
the direction normal to the cracks at a frequency of $100 \mathrm{kHz}$.

First, we determine the background properties from the P- and S-waves velocities for the unfractured dry rock given by Rathore et al. (1995), $V_{p}=2.529 \mathrm{~km} / \mathrm{s}, V_{s}=1.558$ $\mathrm{km} / \mathrm{s}$ at $f=100 \mathrm{kHz}$. Some parameters of interest were measured for the water-saturated sample, such as, $\rho_{b}=1.712 \mathrm{~kg} / \mathrm{m}^{3}, \phi=0.346, K_{f}$ (water $)=2.16$ GPa. Assuming that, under dry conditions, we can approximate $K_{f}($ air $)=0.0001 \mathrm{GPa}$ and $\rho_{f}($ air $)=0.01 \mathrm{~kg} / \mathrm{m}^{3}$, and using the definitions of the elastic velocities

$$
\begin{aligned}
& V_{p}=\sqrt{\frac{L}{\rho_{b}}}, \\
& V_{s}=\sqrt{\frac{\mu}{\rho_{b}}}
\end{aligned}
$$

where $L=K_{m}+\frac{4 \mu}{3}$ is the dry P-wave modulus and

$$
\rho_{b}=\rho_{f} \phi+\rho_{s}(1-\phi)
$$

is the bulk density of the rock, we obtained background parameters. The corresponding dry elastic moduli for the unfractured background turned out to be $K_{m}=4.324 \mathrm{GPa}, \mu_{m}=3.326$ GPa and from Equation 11 the effective solid grain density of the quartz-epoxy matrix is $\rho_{s}=2.088 \mathrm{~kg} / \mathrm{m}^{3}$.

Next, the fracture parameters are computed from the measured P- and S-wave velocities at incidence angles of $11.25^{\circ}$ and $101.25^{\circ}$ with respect to the normal to the crack planes. The velocities are $V_{p}^{d r y}\left(11.25^{\circ}\right)=1.78 \mathrm{~km} / \mathrm{s}, V_{p}^{d r y}\left(101.25^{\circ}\right)=2.472 \mathrm{~km} / \mathrm{s}, V_{s h}^{d r y}\left(11.25^{\circ}\right)=1.26$ $\mathrm{km} / \mathrm{s}$, and $V_{s h}^{d r y}\left(101.25^{\circ}\right)=1.5 \mathrm{~km} / \mathrm{s}$. For the fractures, we assume a very high porosity $\left(\phi_{f}=0.9\right)$ and the same properties at the grain-scale, that is, $\rho_{s}$ and $K_{s}$, as the background. Following the estimations of Brown and Gurevich (2004), the bulk modulus of the grain material is fixed to $30 \mathrm{GPa}$, as it is reasonable for a sand-epoxy matrix. The planar fractures are separated by $2 \mathrm{~mm}$, which is equal to the separation between crack planes considered 
by Rathore et al. (1995). However, due to the differences in the geometry of the fractures between the synthetic sample and the model, we allow the aperture of the planar fractures $H_{f}$ of the model to be different to that of the penny-shaped cracks of the synthetic sample.

Using these parameters, we follow a minimization process to obtain the rest of the fracture properties, that is, $\mu_{m}, K_{m}$, and $H_{f}$. To do so, we compute the effective $C_{i j}$ coefficients in dry conditions using the Backus averaging technique. It is important to note that, without loss of generality and since the measurements were made in a way that the medium has effective vertical TI (VTI), we have to rotate the coordinates system to get a correct interpretation of the determined velocities and stiffness components. Finally, the phase velocity curves as functions of angles of incidence that need to be compared with the measurements can be computed using Equations B-3 to B-5 (Appendix B).

By performing the optimization procedure using the package fminsearchcon developed for Matlab (D'Errico, 2006), the elastic moduli for the planar fractures turned out to be $\mu_{m}=0.0617 \mathrm{GPa}$ and $K_{m}=0.00548 \mathrm{GPa}$, and the fractures aperture $H_{f}=0.022 \mathrm{~mm}$. We have verified the convergence of the optimization procedure by considering multiple initial estimates. Notice that the obtained aperture is remarkably similar to the aperture of the penny-shaped cracks in the synthetic sample (Rathore et al., 1995). Given that fracture apertures tend to vary from very thin $(0.001-0.01 \mathrm{~mm})$ to relatively wide $(0.1-0.5 \mathrm{~mm})$ (Bakulin et al., 2000), this aperture can be thought as a representative value.

The experimental and numerical phase velocities as functions of incidence angle under dry conditions are shown in Figure 2a. The agreement between the numerical velocities and the experimental data is very good, which implies that the dry fracture and background parameters obtained are reasonable and that, as expected, the two models are comparable 


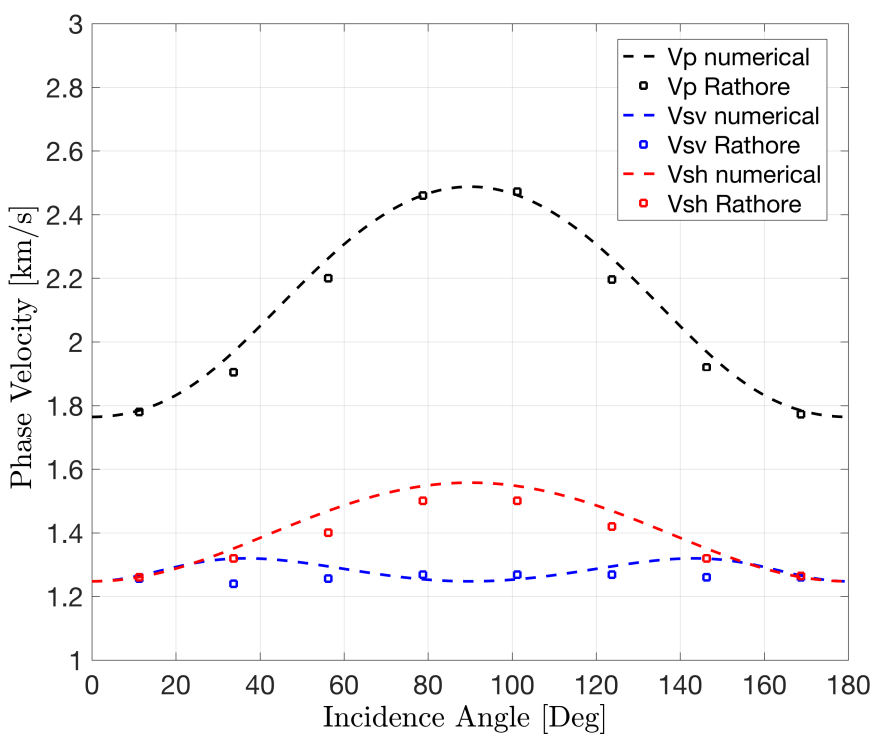

(a)

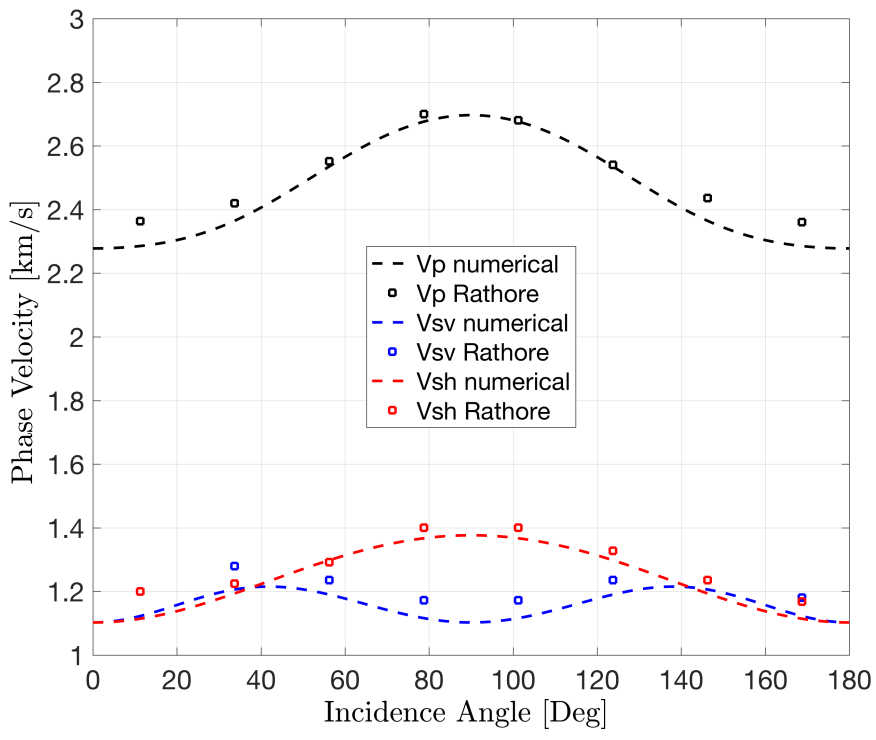

(b)

Figure 2: Comparison of the data from Rathore et al. (1995) (symbols) for (a) dry and (b) water-saturated fractured sandstone with the corresponding best-fitting planar fractures model (dashed lines). 
for dry conditions. These results further validate the conjecture that a plane distribution of small cracks can be replaced by an equivalent planar fracture of constant aperture with appropriate infill material (Hudson and Liu, 1999). In the case of fluid-saturated samples, it is necessary to define the permeabilities of the fractures and of the background. For the fractures, we assume a high permeability (100 D) whereas the background permeability is known and equal to 11.4 D (Brown and Gurevich, 2004), which is representative of a synthetic high-porosity sandstone composed of clean, coarse-grained and well-sorted sand. Moreover, the relatively high permeability reported by Brown and Gurevich (2004) is in qualitative agreement with Maultzsch et al. (2003), who found that the frequency of the measurements is in the low-frequency regime with respect to the characteristic frequency of the effects of WIFF between the cracks and the background. Figure $2 \mathrm{~b}$ shows the Pand S-wave velocities for the water-saturated sample. The overall agreement between the numerical and the experimental velocities is reasonably good. However, there are some differences in the magnitude of the anisotropy. These are expected for several reasons, such as, (i) the fact that we compute the model parameters from the measurements under dry conditions without any input from the velocities in the saturated sample; (ii) the fluid pressure diffusion patterns of a fluid-saturated rock containing infinite fractures and finitesize cracks are different (Wenzlau et al., 2010; Guo et al., 2017); (iii) we assume that the frequency of the measurements is lower than the frequency at which scattering and Biot's global flow effects arise; (iv) low intrinsic background anisotropy as a consequence of the manufacturing process (Tillotson et al., 2011) is neglected.

Lastly, from the set of parameters obtained, it is possible to compute the dry normal $Z_{N}$ and tangential $Z_{T}$ compliances of the sample. The compliances are defined as (Schoenberg 
and Sayers, 1995)

$$
\begin{aligned}
& Z_{N}=\frac{1}{H} \sum_{i=1}^{N^{f}} \frac{H_{f}^{i}}{L_{f}^{i}}, \\
& Z_{T}=\frac{1}{H} \sum_{i=1}^{N^{f}} \frac{H_{f}^{i}}{\mu_{f}^{i}},
\end{aligned}
$$

where $L_{f}^{i}$ and $\mu_{f}^{i}$ are the dry P-wave modulus and shear modulus of the ith fracture, and $N^{f}$ is the number of fractures in the sample. As fractures are assumed to have the same filling material and aperture, the corresponding normal and tangential compliances of the sample are $Z_{N}=\frac{H_{f}}{H L_{f}}=1.25 \times 10^{-10} 1 / \mathrm{Pa}$ and $Z_{T}=\frac{H_{f}}{H \mu_{f}}=1.78 \times 10^{-10} 1 / \mathrm{Pa}$, respectively. In terms of the individual compliances of the fractures, the dry normal $\left(\eta_{N}=\frac{H_{f}}{L_{f}}\right)$ and tangential $\left(\eta_{T}=\frac{H_{f}}{\mu_{f}}\right)$ compliances are $2.5 \times 10^{-13} \mathrm{~m} / \mathrm{Pa}$ and $3.56 \times 10^{-13} \mathrm{~m} / \mathrm{Pa}$, respectively, which fall into the range of realistic values compiled from laboratory and field data estimations by Worthington and Lubbe (2007).

\section{FRACTURE CLUSTERING EFFECTS ON WIFF}

The frequency dependence of the anisotropic seismic response in the limiting cases of regular and randomly distributed fractures in an infinite medium has been exhaustively studied (e.g. Brajanovski et al., 2005; Gurevich et al., 2009). However, current analytical models cannot account for the seismic effects of the clustering of fractures as a result of nonuniform fracture distributions. In this section, we numerically analyze how the presence of an effective additional length scale given by the clustering of fractures affects the effective frequency-dependent seismic anisotropy of the medium. We initially focus on the P-wave modulus normal to the fractures as it presents the largest variability of the seismic response due to the presence of fractures and associated WIFF effects.

It is well known that the frequency range at which the effects of WIFF between the 
fractures and background (FB-WIFF) are maximal is related to the background permeability (Brajanovski et al., 2006). Given that reservoirs typically have permeabilities much lower than that of the synthetic sample of Rathore et al. (1995), we chose a relatively low background permeability of $2 \times 10^{-5} \mathrm{D}$ for the numerical analysis. This in turn shifts the FB-WIFF characteristic frequency to the seismic frequency range $(\sim 100 \mathrm{~Hz})$. Unless indicated otherwise, we use in the following the elastic and hydraulic parameters given in Table 1 for the analysis of WIFF effects on the anisotropic seismic properties of a rock containing vertically aligned fractures.

\begin{tabular}{lll}
\hline Property & Background & Fracture \\
\hline Porosity $\phi$ & 0.346 & 0.9 \\
Permeability $\kappa$ & $20 \mu \mathrm{D}$ & $100 \mathrm{D}$ \\
Solid grain bulk modulus $K_{s}$ & $30 \mathrm{GPa}$ & $30 \mathrm{GPa}$ \\
Frame bulk modulus $K_{m}$ & $4.324 \mathrm{GPa}$ & $0.00548 \mathrm{GPa}$ \\
Frame shear modulus $\mu_{m}$ & $3.326 \mathrm{GPa}$ & $0.0617 \mathrm{GPa}$ \\
Solid grain density $\rho_{s}$ & $2088 \mathrm{~kg} / \mathrm{m}^{3}$ & $2088 \mathrm{~kg} / \mathrm{m}^{3}$ \\
Fluid density $\rho_{f}$ & $1090 \mathrm{~kg} / \mathrm{m}^{3}$ & $1090 \mathrm{~kg} / \mathrm{m}^{3}$ \\
Fluid shear viscosity $\eta_{f}$ & $0.01 \mathrm{Poise}$ & $0.01 \mathrm{Poise}$ \\
Fluid bulk modulus $K_{f}$ & $2.16 \mathrm{GPa}$ & $2.16 \mathrm{GPa}$ \\
\hline
\end{tabular}

Table 1: Physical properties of the materials employed in the numerical analysis. 


\section{General characteristics of the seismic signatures of fracture clusters}

In order to analyze the most general aspects of the effects of fracture clustering on seismic wave propagation, we first consider the arguably simplest representation of a cluster of fractures given by a periodic alternation of regions characterized by a large number of parallel fractures and unfractured background material. The total thickness of such a cluster is typically much larger than that of the individual fractures. For simplicity, we assume that the fractures have a constant aperture and compliance, and that they are regularly distributed inside the cluster.
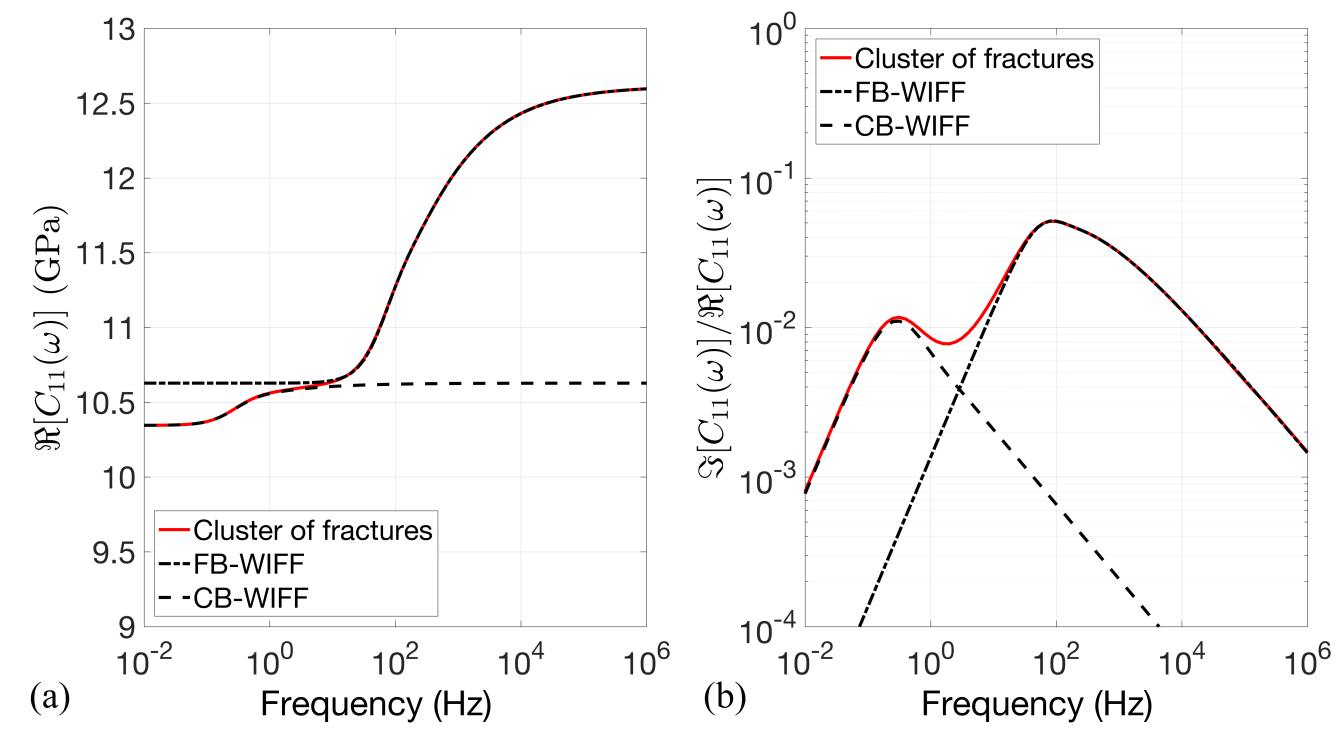

Figure 3: (a) Real part and (b) ratio between imaginary and real parts of $C_{11}(\omega)$ as functions of frequency for a medium containing regularly distributed clusters of fractures (solid line). For comparison, we include the cases of regularly distributed fractures (dashdot line), and regularly distributed layers with effective properties similar to those of the clusters (dashed line). 
The elastic and hydraulic material properties are those given in Table 1 and we assume that each cluster contains 10 regularly distributed fractures with a distance of $H_{b}=2 \mathrm{~mm}$ between consecutive fractures. The distance between consecutive clusters of fractures is chosen to be $10 \times H_{b}=2 \mathrm{~cm}$. Figure 3 shows $C_{11}(\omega)$ and the corresponding attenuation as functions of frequency. This corresponds to the case of a P-wave propagating normal to the fractures. We observe the presence of three regimes for which the real component of $C_{11}(\omega)$ is virtually constant separated by two regimes of maximal dispersion. Correspondingly, the attenuation, which can be quantified by $\Im\left[C_{11}(\omega)\right] / \Re\left[C_{11}(\omega)\right]$, exhibits two peaks.

In order to better understand the frequency dependence of $C_{11}(\omega)$, we include in Figure 3 the effective P-wave modulus of two other media. First, we consider a medium with the same background material and the same number of fractures per unit length but assuming a regular distribution of fractures. In Figure 3, we refer to this model as FB-WIFF, that is, fracture to background WIFF. The second model that we consider is composed by an alternation of two relatively thick layers. A background layer whose thickness is equal to the distance between fracture clusters and another layer whose properties are equivalent to those of the cluster. The effective poroelastic properties of the layer representing the cluster of aligned fractures are computed following Milani et al. (2016), who give general expressions for layered media. In Figure 3, we refer to this model as CB-WIFF, that is, cluster to background WIFF. For the two above mentioned models, the effective P-wave modulus can be computed using the two-layer model of White et al. (1975)

$$
\frac{1}{C_{11}(\omega)}=\left\langle\frac{1}{L^{u}}\right\rangle+\frac{2}{\sqrt{i \omega \eta} H} \frac{\left(\frac{\alpha_{1} M_{1}}{L_{1}^{u}}-\frac{\alpha_{2} M_{2}}{L_{2}^{u}}\right)^{2}}{\sqrt{\frac{L_{1} M_{1}}{L_{1}^{u} \kappa_{1}}} \cot \left(\sqrt{\frac{i \omega \eta L_{1}^{u}}{\kappa_{1} M_{1} L_{1}}} \frac{H_{1}}{2}\right)+\sqrt{\frac{L_{2} M_{2}}{L_{2}^{u} \kappa_{2}}} \cot \left(\sqrt{\frac{i \omega \eta L_{2}^{u}}{\kappa_{2} M_{2} L_{2}}} \frac{H_{2}}{2}\right)},
$$

where $L^{u}=\lambda+2 \mu$ is the undrained P-wave modulus, $H=H_{1}+H_{2}$, and the subscripts 1 
and 2 refer to the different layers.

Let us start by comparing the response of the numerical model with the model containing regularly distributed fractures. In Figure 3, we observe that the FB-WIFF model perfectly explains the intermediate- and high-frequency regimes of the numerically computed $C_{11}(\omega)$. This implies that the attenuation peak observed at higher frequencies is related to WIFF between the fractures and the background inside the clusters. It is important to mention that in order to have the same magnitude and frequency dependence as the FB-WIFF model, we have used $H_{1}=2 \mathrm{~mm}$ in Equation 13. This corresponds to the separation of the fractures inside the cluster, while $H$ was given by the inverse of the fracture intensity in the whole sample. The agreement between the low-frequency limit of the FB-WIFF model and the intermediate regime of the numerical model implies that defining $H_{1}$ in Equation 13 as the distance between fractures in the cluster is equivalent to allowing the fluid pressure of the fractures to equilibrate inside the cluster but not with respect to the rest of the background. This comparison suggests that both fracture density and fracture spacing variations affect the effective properties in this regime.

The modulus dispersion and attenuation observed at low frequencies can be perfectly described by the CB-WIFF model (Figure 3). This, in turn, implies that the modulus dispersion and attenuation in this regime mainly depend on the contrast between the unfractured background and the effective layer representing the cluster of fractures. We have named this regime the CB-WIFF regime, as it is related to the fluid pressure diffusion between the clusters of fractures and the background. Figure 3 shows that the low-frequency limit of the fractured medium coincide with that of the CB-WIFF model. At these frequencies, regardless of the fracture distributions, the whole rock is in fluid pressure equilibrium and its response can be described by the poroelastic Backus average under relaxed con- 
ditions. The high-frequency limit of the CB-WIFF model, on the other hand, coincides with the intermediate regime of the model with clusters of fractures. At these intermediate frequencies, there is no time for fluid pressure equilibration between the cluster as a whole and the background but the fractures remain in pressure equilibrium inside the cluster. Correspondingly, this limit coincides with the low-frequency limit of the FB-WIFF model. Notice that the second dispersion regime cannot be accounted for by the CB-WIFF model as the stiffening effect of the fracture fluid with frequency is missing when representing a cluster of fractures with an effective poroelastic layer.

It is possible to further explore the regions where FB- and CB-WIFF are occurring through the plot of the local contribution to the inverse quality factor per unit area (Solazzi et al., 2016). Following Rubino et al. (2016), we refer to this characteristic as the inverse quality factor density. Figure 4 shows the inverse quality factor density along the sample for frequencies between $1 \times 10^{-4} \mathrm{~Hz}$ and $1 \times 10^{6} \mathrm{~Hz}$. For a given frequency, the regions where energy dissipation due to WIFF takes place are characterized by a larger inverse quality factor density. We observe that at low frequencies the attenuation is negligible. The same scenario prevails in the high-frequency limit, where the fluid has no time to move between pores in the background and the fractures during a half-wave cycle and, hence, fluid flow effects become negligible. This is illustrated by the decreasing distance of fluid diffusion into the background towards higher frequencies. The quality factor density shows that the energy dissipation in the background is maximal at the two frequencies where the attenuation in Figure 3 reaches its maximum values. At $f_{C B}=\omega_{C B} / 2 \pi \sim 0.3 \mathrm{~Hz}$, fluid flow occurs mainly from the cluster of fractures to the unfractured region between clusters. Conversely, at $f_{F B}=\omega_{F B} / 2 \pi \sim 100 \mathrm{~Hz}$, fluid flow prevails in the vicinity of the fractures inside the clusters. 

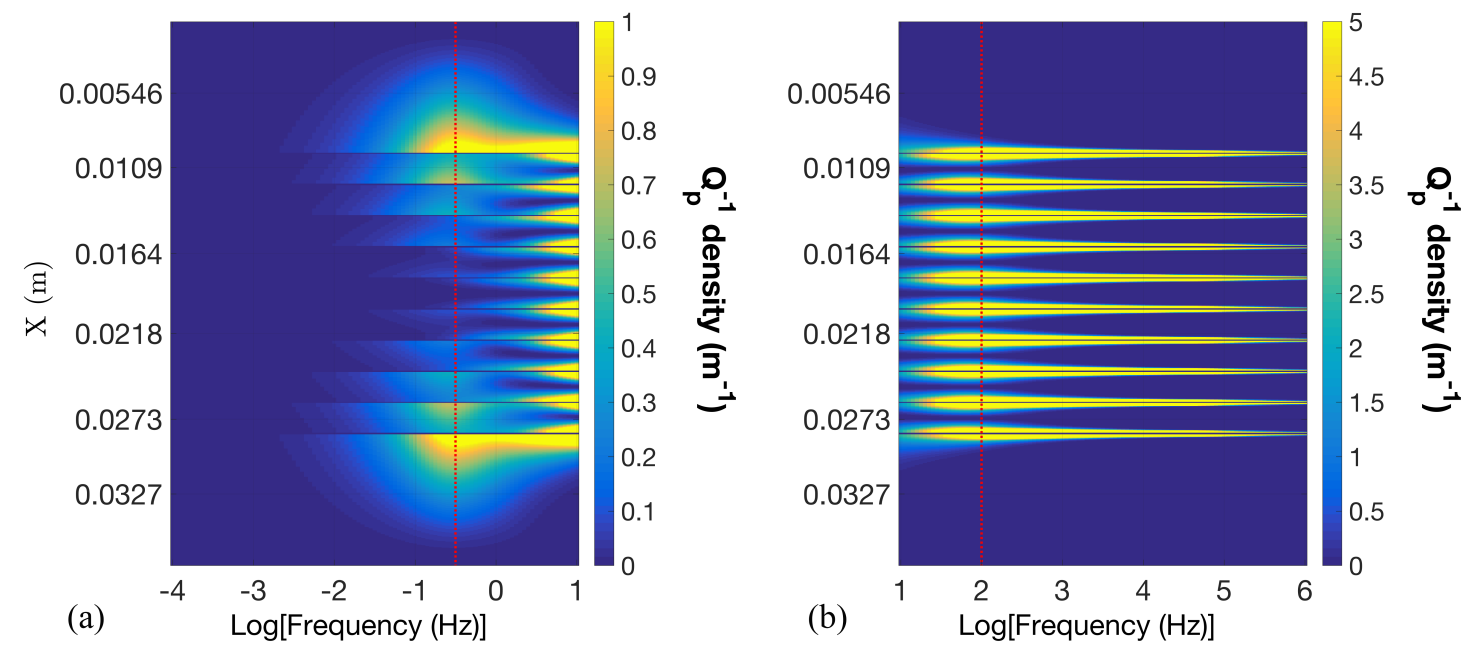

Figure 4: Inverse quality factor density along the sample for frequencies between $1 \times 10^{-4}$ $\mathrm{Hz}$ and $1 \times 10^{6} \mathrm{~Hz}$. Left and right panels correspond to the frequency ranges at which (a) CB-and (b) FB-WIFF dominate. The two red vertical lines correspond to the frequencies where the attenuation of $\mathrm{P}$-waves propagating normal to the fractures has a local maximum.

Lastly, in Figure 5 we illustrate the impact of the geometry and distribution of the clusters on the WIFF effects observed in the effective P-wave modulus of the sample. We consider different sizes of clusters (10 and 100 fractures) and different distances between clusters $\left(H_{c}=10 \times H_{b}\right.$ and $\left.H_{c}=100 \times H_{b}\right)$. In all cases, the distance between fractures inside the cluster is equal to $H_{b}=2 \mathrm{~mm}$ (Table 2 ).

We observe that the FB-WIFF characteristic frequency coincides for the three scenarios, that is, regardless of the intensity of fractures. This is an unexpected result, as analytical models predict a direct dependence of this frequency with the intensity of fractures. Gurevich et al. (2009) shows that the characteristic frequency $\omega_{F B}$ is the inverse of the 

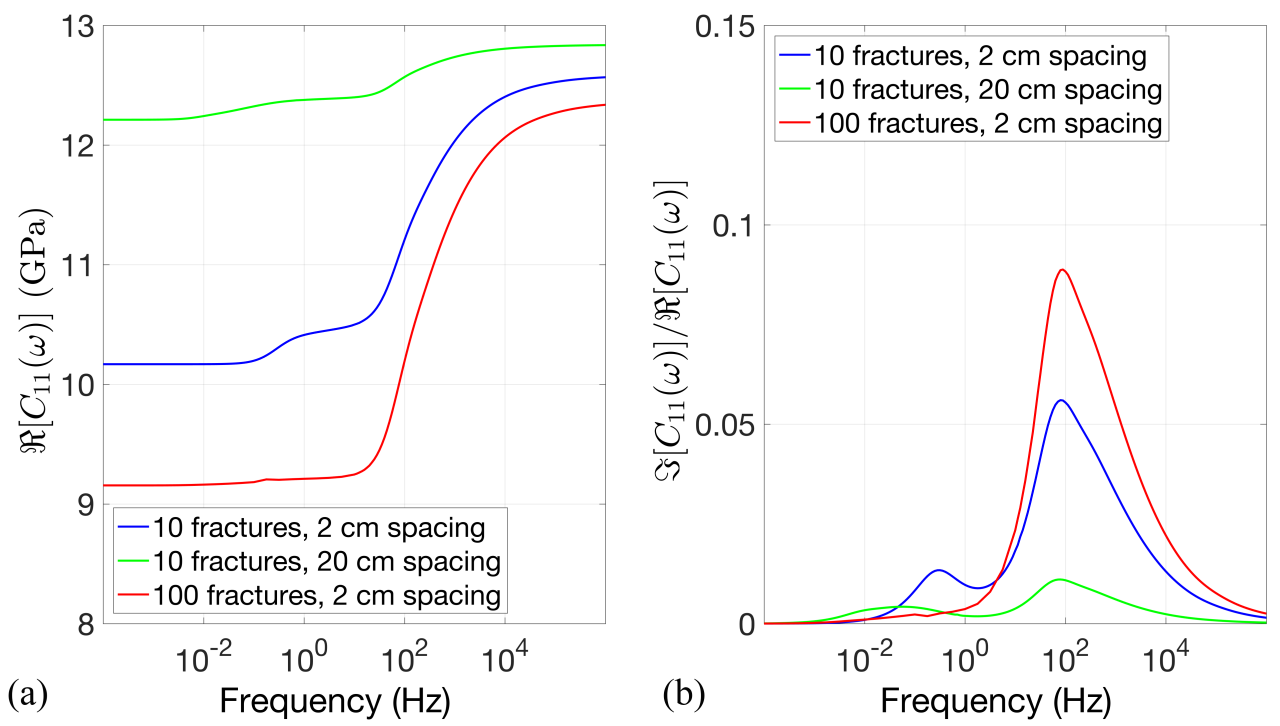

Figure 5: (a) Real part and (b) ratio between imaginary and real parts of $C_{11}(\omega)$ for clusters of 100 fractures separated $2 \mathrm{~cm}$ (red lines), clusters of 10 fractures separated $2 \mathrm{~cm}$ (blue lines), and clusters of 10 fractures separated $20 \mathrm{~cm}$ (green lines). In all cases, the distance between adjacent fractures is $2 \mathrm{~mm}$.

characteristic time of the fluid pressure diffusion process between the fractures and the background. The model of Guo et al. (2017), which accounts for the finite thickness of fractures, allows to estimate this characteristic time $\tau_{F B}$ for the case of aligned fractures

$$
\tau_{F B}=\left(\frac{C_{11}^{u}-C_{11}^{r}}{C_{11}^{r} G}\right)^{2}
$$

with $C_{11}^{r}$ and $C_{11}^{u}$ being the low- and high- frequency limits of $C_{11}(\omega)$ and

$$
G=\frac{\frac{2}{H} C_{11}^{u}\left(\frac{\alpha_{1} M_{1}}{L_{1}^{u}}-\frac{\alpha_{2} M_{2}}{L_{2}^{u}}\right)^{2}}{\sqrt{\frac{M_{1} L_{1} \eta}{L_{1}^{u} \kappa_{1}}}+\sqrt{\frac{M_{2} L_{2} \eta}{L_{2}^{u} \kappa_{2}}}} .
$$

In Equation 15 the subscripts 1 and 2 refer to the background and fracture properties, respectively. Notice that, according to Equation 15, the only information regarding the 


\begin{tabular}{llll}
\hline Parameters & Case 1 & Case 2 & Case 3 \\
\hline$N_{f}$ & 10 & 100 & 10 \\
$H_{b}$ & $2 \mathrm{~mm}$ & $2 \mathrm{~mm}$ & $2 \mathrm{~mm}$ \\
$H_{c}$ & $2 \mathrm{~cm}$ & $2 \mathrm{~cm}$ & $20 \mathrm{~cm}$ \\
$H$ & $3.8 \mathrm{~mm}$ & $2.2 \mathrm{~mm}$ & $21.8 \mathrm{~mm}$ \\
\hline
\end{tabular}

Table 2: Geometrical characteristics of the fracture clusters.

fracture distribution involved in the computation of $\omega_{F B}$ is the mean spacing between fractures which, in turn, defines $H$. This is a consequence of the assumption of low fracture density in the model of Guo et al. (2017), which implies that $H_{b} \gg H_{f}$. According to Equations 14 and 15, the characteristic frequency $f_{F B}=\omega_{F B} / 2 \pi$ for the three scenarios are $f_{F B}^{1} \sim 90 \mathrm{~Hz}, f_{F B}^{2} \sim 117 \mathrm{~Hz}$ and $f_{F B}^{3} \sim 63 \mathrm{~Hz}$. The discrepancies of the analytical prediction with the numerical results imply that the FB-WIFF frequency is mainly controlled by the spacing of the fractures inside the cluster, which in the three cases remains unchanged. The fracture intensity, that is $1 / H$ (Bakulin et al., 2000), controls the magnitude of the FB-WIFF effects, which decrease with lower fracture densities.

Furthermore, we observe that the geometric characteristics of the fracture clusters strongly affect the amount of attenuation and dispersion as well as the characteristic frequency due to CB-WIFF. For clusters containing the same intensity of fractures, the corresponding characteristic frequency shifts towards lower values when the distance between clusters increases (blue and green lines in Figure 5). As mentioned before, at these rela- 
tively low frequencies, a cluster of fractures effectively behaves as a layer. Given that in both models the thickness of the effective layer is the same, the CB-WIFF characteristic frequency depends on the distance between consecutive layers. As this distance increases, the interference of the fluid pressure diffusion in the unfractured background occurs at lower frequencies (Brajanovski et al., 2006). In addition, as the density of clusters per unit length decreases, the level of attenuation decreases as well. Lastly, when the number of fractures in the cluster increases, while keeping the distance between clusters constant, the size of the cluster of fractures gets much larger than the distance between consecutive clusters and the model approaches the limiting case of a regular distribution of fractures. Correspondingly, the effects related to the fracture cluster become negligible, as illustrated by the comparison between blue and red curves in Figure 5.

\section{Fracture clustering effects as a consequence of realistic spacing distribu- tions}

Given that the previous analysis was based on a simplistic representation of fracture clustering, in this section, we aim at analyzing the corresponding impact of CB-WIFF effects when dealing with realistic fracture distributions. In agreement with previous findings (Gillespie et al., 1993; Bonnet et al., 2001), Fang et al. (2016) argue that natural fractures often exhibit a distribution of fracture spacings following a power-law function and that this kind of distribution leads to the formation of clusters of fractures.

Hence, in order to explore the effects of clusters in the case of realistic distributions of fracture spacing, we generate random fracture models following a power-law function. That 
is, we assume that the variable fracture spacing $H_{b}$ is given by (Fang et al., 2016)

$$
H_{b}=\left[H_{\min }^{n}+m \cdot\left(H_{\max }^{n}-H_{\min }^{n}\right)\right]^{1 / n},
$$

where $n$ is the power-law exponent governing the distribution, $m$ is a random number between 0 and 1 , and $H_{\min }$ and $H_{\max }$ are the minimum and maximum values for fracture spacing, respectively.
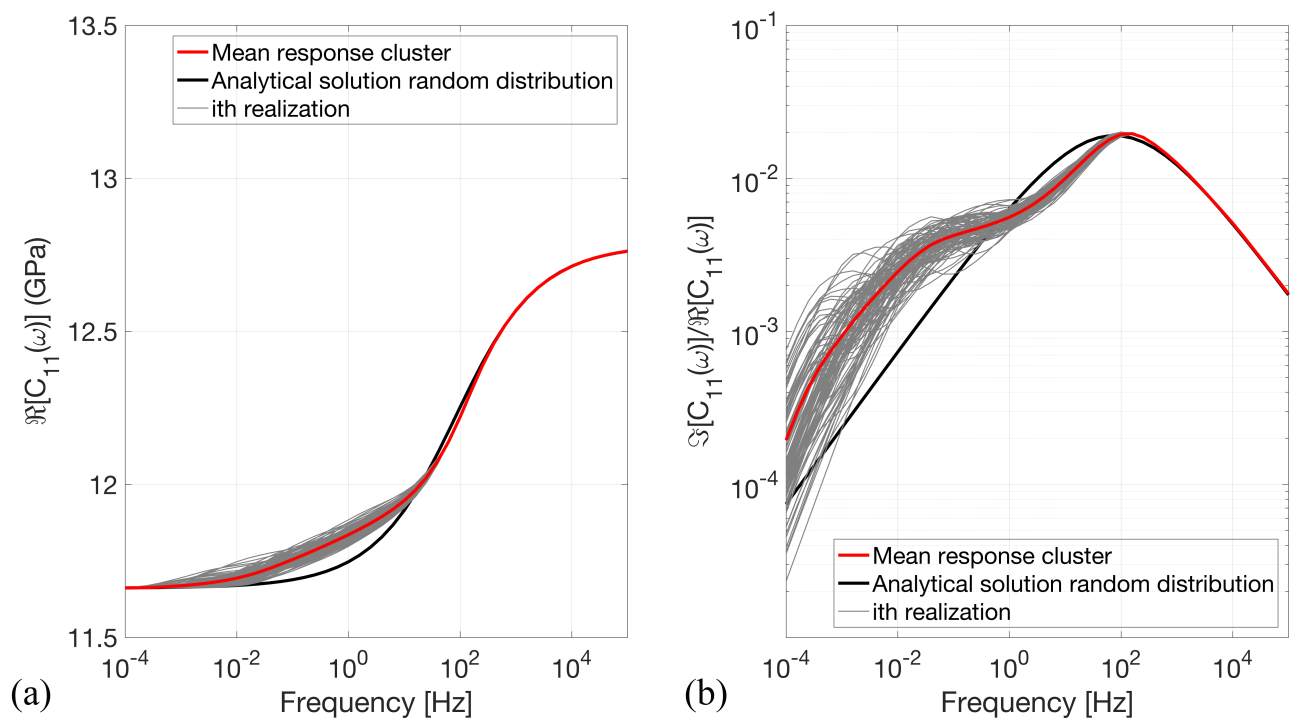

Figure 6: (a) Real part and (b) ratio between imaginary and real parts of $C_{11}(\omega)$ as functions of frequency for 100 samples containing randomly distributed fractures according to case 1 in Table 3 (grey lines). Red curves correspond to the mean values of the realizations. For comparison, we include the analytical solution for a uniform random distribution of fractures with the same fracture intensity (black line).

Figure 6 shows the effective P-wave modulus dispersion and attenuation normal to the fractures for 100 samples containing a random distribution of fractures whose parameters correspond to case 1 in Table 3. For comparison, we include the analytical model of Guo 
et al. (2017) for the P-wave modulus normal to a uniform random distribution of planar fractures. We observe that for samples containing fractures distributed according to Equation 16, the effective P-wave modulus presents two attenuation and dispersion regimes. As expected, the real component of the effective P-wave modulus shows that, at the low-and high-frequency limits, the fracture distribution does not play any role and, hence, both the numerical and analytical models coincide in these limits. However, its frequency dependence is affected by the presence of fracture clustering, producing a deviation from the analytical solution. Regarding the CB-WIFF manifestation in the seismic attenuation, we can identify different low-frequency asymptotes for the numerical and analytical solutions. The former presents an asymptote that is proportional to $f$, while for the latter it is proportional to $\sqrt{f}$.

\begin{tabular}{llll}
\hline Parameters & Case 1 & Case 2 & Case 3 \\
\hline$H_{\text {min }}$ & $0.001 \mathrm{~m}$ & $0.001 \mathrm{~m}$ & $0.005 \mathrm{~m}$ \\
$H_{\max }$ & $0.1 \mathrm{~m}$ & $0.1 \mathrm{~m}$ & $0.05 \mathrm{~m}$ \\
$n$ & -1 & -2 & -1 \\
Total length & $0.5 \mathrm{~m}$ & $0.3 \mathrm{~m}$ & $0.5 \mathrm{~m}$ \\
$N_{f}$ & 50 & 100 & 50 \\
\hline
\end{tabular}

Table 3: Power-law function parameters for the numerical analysis.

Figure 7 shows the effective properties of 100 samples considering cases 2 and 3 of Table 3. In the case of a higher exponent $n$ (case 2), the number of small spacings between fractures compared to large ones increases. This produces a steeper dispersion and, correspondingly, a 

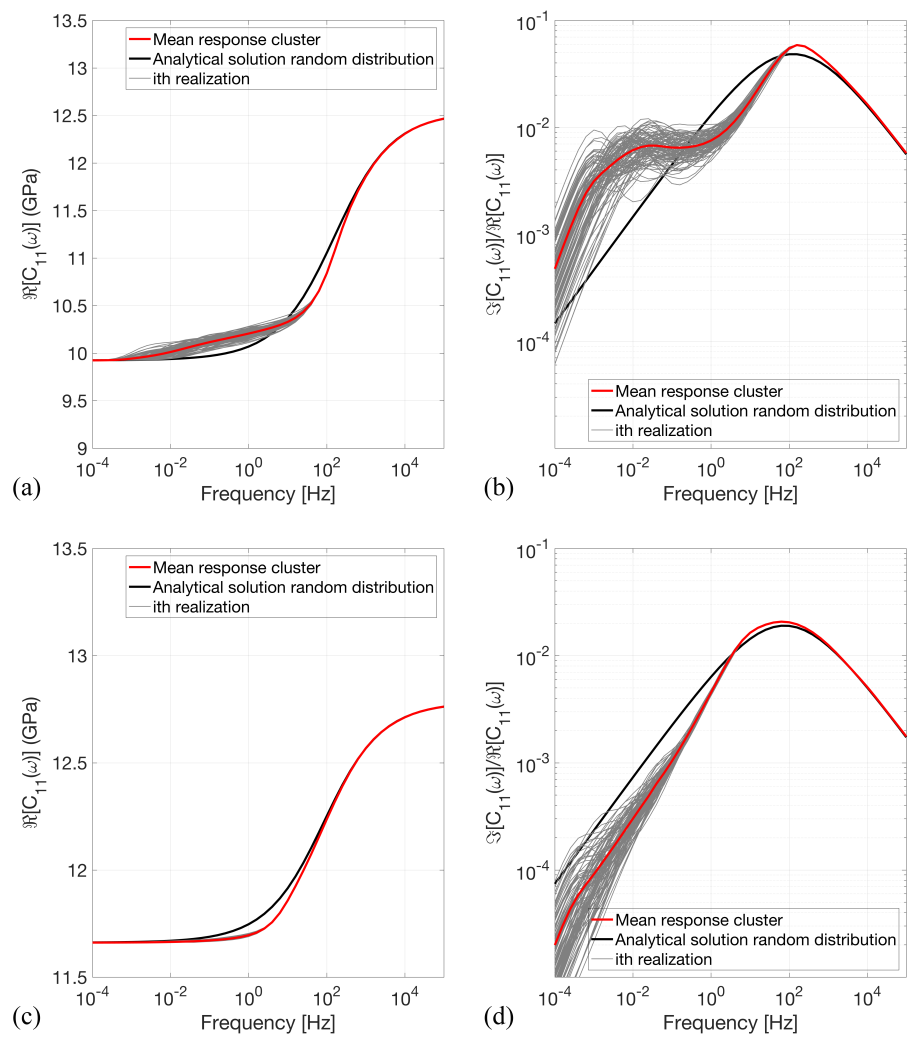

Figure 7: (a, c) Real part and (b, d) ratio between imaginary and real parts of $C_{11}(\omega)$ as functions of frequency for 100 samples containing randomly distributed fractures (grey lines). Top and bottom panels correspond to cases 2 and 3 of Table 3 , respectively. Red curves correspond to the mean values of the realizations. For comparison, we include the analytical solution for a uniform random distribution of fractures with the same fracture intensity (black lines).

narrower seismic attenuation related to FB-WIFF effects compared to the random analytical solution. As shown in the analysis of Figure 5, when a given fracture spacing dominates the distribution, the effective properties in the FB-WIFF regime tend to those of a regular distribution of fractures. Correspondingly, the magnitude of the CB-WIFF effects become 
less significant than those associated with FB-WIFF.

Regarding case 3, the effective response is closer to that of a two-layer model. As mentioned before, the manifestation of the CB-WIFF and its amplitude is related to the thickness of the sections of the sample with closely packed fractures and to the thickness of the adjacent unfractured background. As, in this case, we decrease the range of possible values for the power-law, the spacing between fractures is more homogeneous and the manifestation of CB-WIFF less significant. Hence, although there are some discrepancies at low frequencies, the effective P-wave modulus is well described by the analytical model.

For the analysis of each of the scenarios shown in Figures 6 and 7, we have fixed the number of fractures as well as the total length of the samples. By doing so, we have minimized the dispersion of the realizations with respect to the mean response and focused on the variations due to clustering effects. Although not shown here for brevity, we have performed numerical simulations without restrictions on the total length of the samples, thus allowing for different fracture densities in each realization. The results show that the changes in CB- and FB-WIFF effects coincide with those shown in this section.

\section{Seismic anisotropy analysis}

As mentioned before, our methodology does not only allow us to compute the effective Pwave modulus normal to an arbitrary distribution of aligned fractures but it also provides the effective anisotropic stiffness matrix of the medium. Since the current consensus is that the orientation, density, scale, and spacing of fractures as well as the fluid properties can be extracted from frequency-dependent seismic anisotropy (Bakulin et al., 2000; Tillotson et al., 2011; Liu and Martinez, 2013), we proceed to explore the WIFF effects on seismic 
anisotropy. Although there exist several seismic attributes quantifying seismic anisotropy, for brevity, we focus the analysis on Thomsen's (1986) parameters to quantify the velocity anisotropy and on the formulas of Collet and Gurevich (2016) to quantify the corresponding attenuation parameters. In the particular case of an HTI medium whose effective stiffness matrix is given by Equation 7, the set of anisotropic parameters quantifying the velocity anisotropy are (Bakulin et al., 2000)

$$
\begin{aligned}
\epsilon & =\frac{\left(C_{11}-C_{33}\right)}{2 C_{33}}, \\
\delta & =\frac{\left(C_{13}+C_{66}\right)^{2}-\left(C_{33}-C_{66}\right)^{2}}{2 C_{33}\left(C_{33}-C_{66}\right)}, \\
\gamma & =\frac{\left(C_{66}-C_{44}\right)}{2 C_{44}} .
\end{aligned}
$$

The parameters $\epsilon$ and $\gamma$ are often called the P- and S-wave velocity anisotropy as in the elastic case they are close to the fractional difference between the horizontal and vertical $\mathrm{P}$ - and $\mathrm{S}_{\|}$-wave velocities. The subscript $\|$indicates that we refer to the $\mathrm{S}$-wave polarized parallel to the fractures plane. The parameter $\delta$, on the other hand, quantifies the second derivative of the P-wave phase velocity at vertical incidence. Bakulin et al. (2000) showed that, in the case of a rock containing vertical fractures, the parameters in Equation 17 are negative.

In the case of weak attenuation, the attenuation anisotropy parameters are given by (Collet and Gurevich, 2016)

$$
\begin{aligned}
\epsilon_{Q} & =\frac{1+2 \epsilon}{2}\left(\frac{1}{Q_{11}}-\frac{1}{Q_{33}}\right) \\
\delta_{Q} & =\frac{(1+\delta)}{Q_{13}}+2 \frac{C_{66}}{C_{11}}\left(\frac{1}{Q_{66}}-\frac{1}{Q_{13}}\right)-\frac{1}{Q_{11}},
\end{aligned}
$$

where $Q_{i j}(\omega)=\frac{\Re\left[C_{i j}(\omega)\right]}{\Im\left[C_{i j}(\omega)\right]}$. The parameters $\epsilon_{Q}$ and $\delta_{Q}$ reduce to zero for non-dissipative media or if the attenuation is isotropic. Given that the stiffness coefficients $C_{i j}(\omega)$ are complexvalued, the attenuation anisotropy parameters are complex-valued as well. In the limit of 
weak attenuation, we can compute the real part of the complex anisotropy parameters given in Equation 18 to obtain a measure of the attenuation anisotropy as a function of frequency (Collet and Gurevich, 2016). For brevity and given that the behavior of the velocity and attenuation anisotropy parameters for randomly and regularly distributed aligned fractures or cracks has been thoroughly described before (e. g., Collet and Gurevich, 2016; Guo et al., 2017), we focus on the sensitivity of such parameters to fracture clustering.
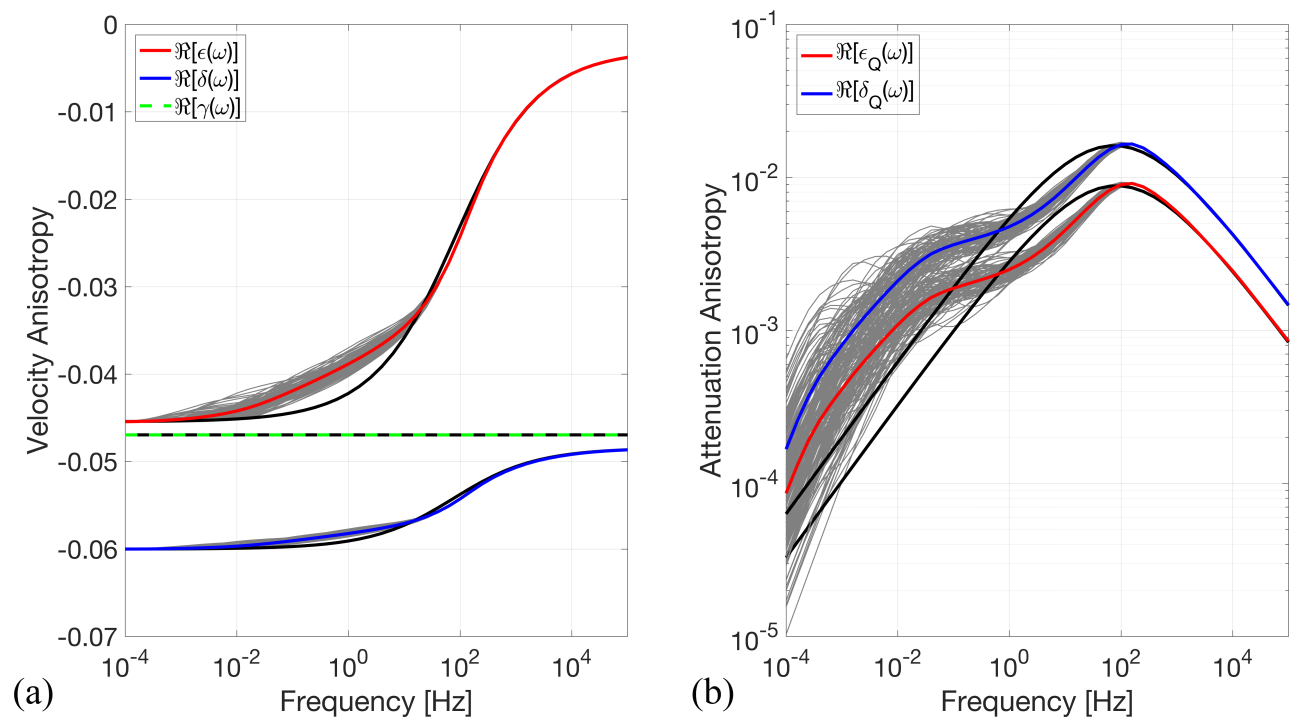

Figure 8: Real components of (a) the Thomsen parameters for velocity and (b) the parameters after Collet and Gurevich (2016) for the attenuation as functions of frequency for 100 samples containing randomly distributed fractures according to case 1 in Table 3 (grey lines). Heavy lines correspond to the mean values of the realizations. For comparison, we also include the analytical solution for a uniform random distribution of fractures with the same fracture intensity (black lines).

Figure 8 shows the velocity and attenuation anisotropy parameters as functions of fre- 
quency for case 1 in Table 3. We include the solution for the analytical model of a random distribution of aligned planar fractures. First, we observe in Figure 8 that $\gamma$ does not change with frequency. The reason for this is that the difference between the velocity of an $\mathrm{S}_{\|}$-wave propagating in the direction normal or parallel to the fractures is controlled by the tangential compliance, which in this case is frequency independent. Hence, there is no evidence of the distribution of the fractures in this parameter.

The parameters $\epsilon$ and $\delta$ are frequency-dependent but only the former reflects the fracture clustering effects. From the definition of $\epsilon$ in Equation 17 it is clear that the behavior of this parameter is controlled by the behavior of $C_{11}(\omega)$, which is strongly affected by CB-WIFF effects. The parameter $\delta$, on the other hand, is almost unaffected by the fractures distribution, which means that the latter has little impact on the shape of the angle dependence of P- and S-waves.

Regarding the attenuation anisotropy parameters, we observe that they follow the same behavior as the attenuation shown in the previous section. They tend to zero in the low- and high-frequency limits and reach their local maxima at both the CB- and FB-WIFF characteristic frequencies. The strong frequency-dependence of $\epsilon_{Q}$ and $\delta_{Q}$ as well as the difference with respect to the random analytical solution indicate that attenuation anisotropy data may be more useful than velocity anisotropy data for characterizing fracture distributions.

\section{CONCLUSIONS}

We have investigated the effects of fracture clustering on the effective seismic properties of porous rocks containing aligned planar fractures. To this end, we developed an hybrid methodology that permits to circumvent the computational challenges associated with per- 
forming 3D numerical relaxation tests and allows us to consider arbitrary distributions of aligned planar fractures. This means that, in addition to variable fracture spacing, which was the main attribute investigated in this work, it also allows to consider arbitrary fracture apertures and elastic and hydraulic contrasts between fractures and background as well as varying fluid properties.

In order to determine the mechanical properties of fractures represented as part of the poroelastic continuum, we used experimental velocities measured on a dry synthetic sandstone containing penny-shaped cracks of known geometry and orientation. We showed that the estimated parameters allow us to reasonably approximate the corresponding velocity anisotropy measurements under fluid-saturated conditions. Using the estimated fracture mechanical characteristics, a corresponding numerical analysis demonstrated that the presence of fracture clustering results in two frequency regimes of seismic attenuation and velocity dispersion. The regime at low frequencies is related to WIFF between the clusters and the background and its characteristic frequency depends on the size and spacing between clusters. The second dispersion and attenuation regime is associated with WIFF between fractures and background inside clusters. Its characteristic frequency is mainly controlled by the fracture intensity inside the cluster rather than by the total fracture intensity. Moreover, we showed that it is possible to quantify both the FB- and CB-WIFF effects using pertinent two-layer poroelastic models. This suggests that it is possible to extend current analytical models to account for fracture clustering by allowing for an additional length scale in the sample.

Analyses of anisotropic parameters allowed us to verify that the velocity anisotropy parameters show little sensitivity to fracture clustering effects. In this case, analytical models of random distributions of planar fractures managed to reproduce most of the frequency- 
dependent effects on the velocity anisotropy parameters. Attenuation anisotropy, on the other hand, turned out to be much more sensitive to fractures distributions.

\section{ACKNOWLEDGMENTS}

This work was supported by a grant from the Swiss National Science Foundation and completed within the Swiss Competence Center for Energy Research-Supply of Electricity with the support of the Swiss Innovation Agency. We wish to thank four anonymous reviewers and the associate editor for the comments and suggestions that helped to improve the quality of this manuscript.

\section{APPENDIX A: RELAXED AND UNRELAXED LIMITS OF THE STIFFNESS TENSOR}

In analogy with the expressions given by Krzikalla and Müller (2011) for the relaxed and unrelaxed limits of the stiffness tensor for VTI media, we give the corresponding expressions for an HTI medium. The Backus formulas for the relaxed limit are

$$
\begin{aligned}
C_{11}^{r} & =\left\langle\frac{1}{L}\right\rangle^{-1}+\frac{Y^{2}}{Z}, \\
C_{12}^{r} & =\left\langle\frac{1}{L}\right\rangle^{-1}\left\langle\frac{\lambda_{d}}{L}\right\rangle+\frac{X Y}{Z}, \\
C_{22}^{r} & =\left\langle\frac{4 \mu\left(\lambda_{d}+\mu\right)}{L}\right\rangle+\left\langle\frac{1}{L}\right\rangle^{-1}\left\langle\frac{\lambda_{d}}{L}\right\rangle^{2}+\frac{X^{2}}{Z}, \\
C_{23}^{r} & =\left\langle\frac{2 \mu \lambda_{d}}{L}\right\rangle+\left\langle\frac{1}{L}\right\rangle^{-1}\left\langle\frac{\lambda_{d}}{L}\right\rangle^{2}+\frac{X^{2}}{Z}, \\
C_{55}^{r} & =\left\langle\frac{1}{\mu}\right\rangle^{-1},
\end{aligned}
$$


where $\langle\psi\rangle=\frac{1}{H} \int \psi(x) d x$ is the poroelastic Backus average, $\lambda_{d}=K_{m}-2 \mu / 3$ is the Lamé constant in dry conditions and the expressions $X, Y$ and $Z$ are given by

$$
\begin{aligned}
& X=-Z\left(\left\langle\frac{2 \alpha \mu}{L}\right\rangle+\left\langle\frac{\alpha}{L}\right\rangle\left\langle\frac{\lambda_{d}}{L}\right\rangle\left\langle\frac{1}{L}\right\rangle^{-1}\right), \\
& Y=-Z\left\langle\frac{\alpha}{L}\right\rangle\left\langle\frac{1}{L}\right\rangle^{-1}, \\
& Z=\left(\left\langle\frac{1}{M}\right\rangle+\left\langle\frac{\alpha^{2}}{L}\right\rangle-\left\langle\frac{\alpha}{L}\right\rangle^{2}\left\langle\frac{1}{L}\right\rangle^{-1}\right)^{-1} .
\end{aligned}
$$

On the other hand, the Backus formulas for the unrelaxed limit are

$$
\begin{aligned}
C_{11}^{u} & =\left\langle\frac{1}{L^{u}}\right\rangle^{-1}, \\
C_{12}^{u} & =\left\langle\frac{1}{L^{u}}\right\rangle^{-1}\left\langle\frac{\lambda_{u}}{L^{u}}\right\rangle, \\
C_{22}^{u} & =\left\langle\frac{4 \mu\left(\lambda_{u}+\mu\right)}{L^{u}}\right\rangle+\left\langle\frac{1}{L^{u}}\right\rangle^{-1}\left\langle\frac{\lambda_{u}}{L^{u}}\right\rangle^{2}, \\
C_{23}^{u} & =\left\langle\frac{2 \mu \lambda_{u} d}{L^{u}}\right\rangle+\left\langle\frac{1}{L^{u}}\right\rangle^{-1}\left\langle\frac{\lambda_{u}}{L^{u}}\right\rangle^{2}, \\
C_{55}^{u} & =\left\langle\frac{1}{\mu}\right\rangle^{-1},
\end{aligned}
$$

where $\lambda_{u}=K_{m}-2 \mu / 3+\alpha^{2} M$ is the Lamé constant in undrained conditions.

\section{APPENDIX B: PHASE VELOCITY AND INVERSE QUALITY FACTOR}

We consider a plane-wave propagating through a homogeneous anisotropic viscoelastic medium. The general plane-wave solution for the displacement vector $\mathbf{u}=\left(u_{x}, u_{y}, u_{z}\right)$ has the form

$$
\mathbf{u}=\mathbf{A} e^{-i \mathbf{k} \cdot \mathbf{r}},
$$

with $\mathbf{k}=\left(k_{1}, k_{2}, k_{3}\right)$ being the wave vector, $\mathbf{A}=[A, B, C]$ the amplitudes of the components of the displacement and $\mathbf{r}=(x, y, z)$ the position vector. Substituting the plane-wave 
solution into the equations of motion in the space-frequency domain

$$
-\omega^{2} \rho \mathbf{u}-\nabla \cdot \boldsymbol{\tau}=0
$$

where $\rho$ is the density of the rock and $\tau$ the stress tensor, and considering the relations between the stress and strain tensors given in Equation 7, we obtain the Kelvin-Christoffel equation (e.g., Carcione, 2007)

$$
\left(\boldsymbol{\Gamma}-\frac{\omega^{2} \rho}{k^{2}} \mathbf{I}_{3}\right) \cdot \mathbf{u}^{T}=0
$$

In this equation, $\Gamma=\mathbf{K} \cdot \mathbf{C} \cdot \mathbf{K}^{T}$ is the symmetric Kelvin-Christoffel matrix with $\mathbf{C}$ given in Equation $7, k$ the wavenumber, and

$$
\mathbf{K}=\left[\begin{array}{cccccc}
k_{1} & 0 & 0 & 0 & k_{3} & k_{2} \\
0 & k_{2} & 0 & k_{3} & 0 & k_{1} \\
0 & 0 & k_{3} & k_{2} & k_{1} & 0
\end{array}\right]
$$

For homogeneous waves, we can write $k_{1}=k \sin (\theta) \cos (\phi), k_{2}=k \sin (\theta) \sin (\phi)$, and $k_{3}=$ $k \cos (\theta)$ with $\theta$ and $\phi$ being the incidence angle with respect to the $x_{3}$ direction and the azimuth angle of the seismic wave, respectively. The solution of the eigenvalue problem of Equation B-3, whose characteristic equation is $\operatorname{det}\left(\boldsymbol{\Gamma}-\frac{\omega^{2} \rho}{k^{2}} \mathbf{I}_{3}\right)=0$, provides the complex wavenumbers for all the possible wave modes.

In an anisotropic medium, three distinct body waves propagate and form an orthogonal polarized set. A P-wave that travels quasi-parallel to the propagation direction and two S-waves that are mutually orthogonal and polarized in the plane perpendicular to the Pwave polarization direction. For practical applications, the S-waves are often distinguished by referring to the faster and slower shear-waves, which are denoted by $S_{1}$ and $S_{2}$.

The three physically meaningful solutions having negative imaginary parts, $k_{P}(\omega, \theta, \phi)$, $k_{S_{1}}(\omega, \theta, \phi)$ and $k_{S_{2}}(\omega, \theta, \phi)$ correspond to the wavenumbers of the $\mathrm{P}_{-}, \mathrm{S}_{1^{-}}$and $\mathrm{S}_{2}$-waves, 
respectively. The corresponding phase velocities and inverse quality factors can be then evaluated as

$$
\begin{aligned}
& V_{j}(\omega, \theta, \phi)=\frac{\omega}{\Re\left(k_{j}(\omega, \theta, \phi)\right)}, \\
& Q_{j}^{-1}(\omega, \theta, \phi)=-\frac{\Im\left(k_{j}^{2}(\omega, \theta, \phi)\right)}{\Re\left(k_{j}^{2}(\omega, \theta, \phi)\right)} \quad \text { for } j=P, S_{1}, S_{2} .
\end{aligned}
$$

\section{APPENDIX C: SINGLE RELAXATION FUNCTION FOR FRACTURE CLUSTERS}

Krzikalla and Müller (2011) demonstrated the correctness of the relation given in Equation 8 for a medium containing regularly distributed fractures. In addition, they considered two rather simple cases, (i) a homogeneous background and (ii) a background composed by two layers with different permeabilities. With our methodology, we can further generalize the validation of the single relaxation function assumption that leads to Equation 8 by considering a rock containing clusters of fractures. As shown in Section "Numerical Analysis", when a rock contains clusters of fractures, there is an additional characteristic length in the sample that, in turn, produces an additional characteristic frequency of the relaxation function. That is, in addition to the relaxed and unrelaxed regimes, there is an intermediate regime for which the medium essentially behaves as a two layer medium composed by the background and an effective layer representing the cluster of fractures.

For the validation, we implement 3D relaxation tests extending the methodology developed by Rubino et al. (2016) and originally proposed for 2D samples. We refer the reader to this work for details on the methodology to obtain the effective stiffness tensor of a poroelastic heterogeneous sample. For the numerical comparison, we use the fracture and

background properties in Table 1. Each cluster of fractures contains three fractures of 0.02 mm thickness, separated by $2 \mathrm{~mm}$. The distance between clusters is $20 \mathrm{~mm}$. 

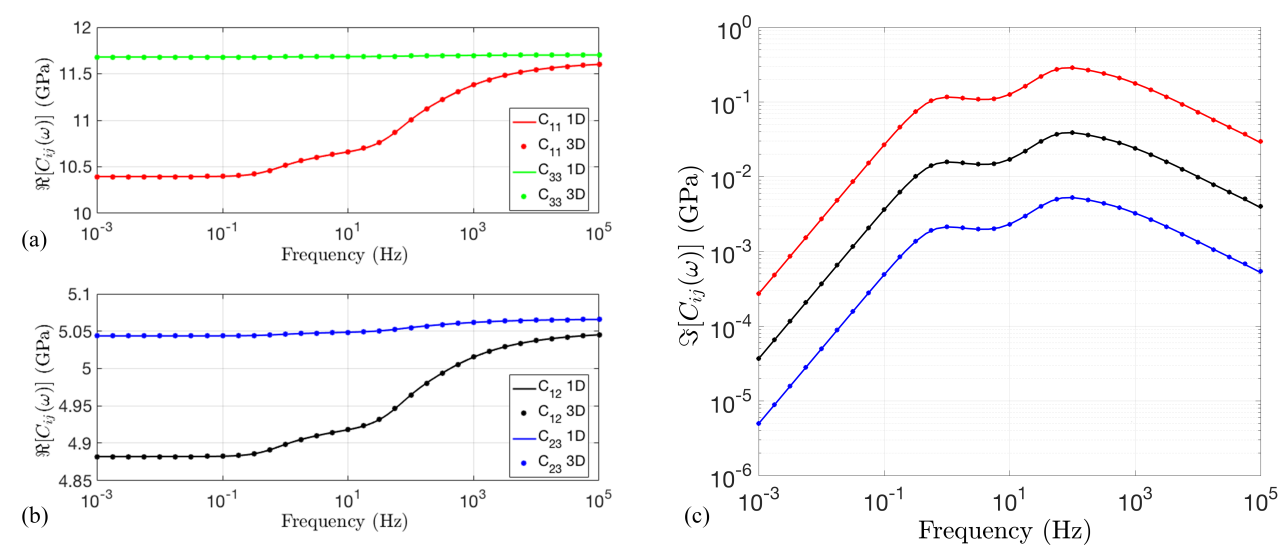

Figure C.1: (a and b) Real and (c) imaginary parts of the effective stiffness coefficients as functions of frequency computed using the hybrid methodology (solid lines) and 3D numerical relaxation tests (dots).

Once the $C_{i j}$ coefficients are computed for the 3D sample, we can compare them with those obtained by following the approach presented in this work (Equations 5 to 8). Figure C.1 shows the comparison between the stiffness coefficients as functions of frequency. The agreement between the two approaches is excellent. Interestingly, this means that the presence of an intermediate regime does not change the relation given in Equation 8 and we only need to know the relaxed and unrelaxed limits and the scalar function $R(\omega)$ to describe the frequency-dependent $C_{i j}$ coefficients. 


\section{REFERENCES}

Bakulin, A., V. Grechka, and I. Tsvankin, 2000, Estimation of fracture parameters from reflection seismic data-Part I: HTI model due to a single fracture set: Geophysics, 65, 1788-1802.

Biot, M. A., 1962, Mechanics of deformation and acoustic propagation in porous media: Journal of Applied Physics, 33, 1482-1498.

Bonnet, E., O. Bour, N. E. Odling, P. Davy, I. Main, P. Cowie, and B. Berkowitz, 2001, Scaling of fracture systems in geological media: Reviews of geophysics, 39, 347-383.

Brajanovski, M., B. Gurevich, and M. Schoenberg, 2005, A model for P-wave attenuation and dispersion in a porous medium permeated by aligned fractures: Geophysical Journal International, 163, 372-384.

Brajanovski, M., T. M. Müller, and B. Gurevich, 2006, Characteristic frequencies of seismic attenuation due to wave-induced fluid flow in fractured porous media: Geophysical Journal International, 166, 574-578.

Brown, L., and B. Gurevich, 2004, Frequency-dependent seismic anisotropy of porous rocks with penny-shaped cracks: Exploration Geophysics, 35, 111-115.

Carcione, J. M., 2007, Wave fields in real media: Wave propagation in anisotropic, anelastic, porous and electromagnetic media: Elsevier, Vol. 38.

Carcione, J. M., B. Gurevich, J. E. Santos, and S. Picotti, 2013, Angular and frequencydependent wave velocity and attenuation in fractured porous media: Pure and Applied Geophysics, 170, 1673-1683. 
Collet, O., and B. Gurevich, 2016, Frequency dependence of anisotropy in fluid saturated rocks-Part I: aligned cracks case: Geophysical Prospecting, 64, 1067-1084.

D'Errico, J., 2006, Fminsearchcon. MATLAB Central File Exchange (http://www. mathworks. com/matlabcentral/fileexchange).

Fang, X, Y. Zheng, and M. C. Fehler, 2016, Fracture clustering effect on amplitude variation with offset and azimuth analyses: Geophysics, 82, N13-N25.

Gale, J. F., S. E. Laubach, J. E. Olson, P. Eichhubl, and A. Fall, 2014, Natural fractures in shale: A review and new observations: AAPG bulletin, 98, 2165-2216.

Galvin, R. J., and B. Gurevich, 2015, Frequency-dependent anisotropy of porous rocks with aligned fractures: Geophysical Prospecting, 63, 141-150.

Gassmann, F., 1951, Über die elastizität poröser medien: Vier. der Natur. Gesellschaft Zürich, 96, 1-23.

Gelinsky, S., and S. A. Shapiro, 1997, Poroelastic Backus averaging for anisotropic layered fluid-and gas-saturated sediments: Geophysics, 62, 1867-1878.

Gillespie, P. A., C. B. Howard, J. J. Walsh, and J. Watterson, 1993, Measurement and characterisation of spatial distributions of fractures: Tectonophysics, 226, 113-141.

Guo, J., J. G. Rubino, N. D. Barbosa, S. Glubokovskikh, and B. Gurevich, 2017, Seismic dispersion and attenuation in saturated porous rocks with aligned fractures of finite thickness: theory and numerical simulations - Part I: P-wave perpendicular to the fracture plane: Accepted for publication in Geophysics.

Gurevich, B., 2003, Elastic properties of saturated porous rocks with aligned fractures: Journal of Applied Geophysics, 54, 203-218. 
Gurevich, B., M. Brajanovski, R. J. Galvin, T. M. Müller, and J. Toms-Stewart, 2009, $\mathrm{P}$-wave dispersion and attenuation in fractured and porous reservoirs-poroelasticity approach: Geophysical Prospecting, 57, 225-237.

Huang, Q., and J. Angelier, 1989, Fracture spacing and its relation to bed thickness: Geological Magazine, 126, 355-362.

Hudson, J. A., 1980, Overall properties of a cracked solid: Mathematical Proceedings of the Cambridge Philosophical Society: Cambridge University Press, 88, 371-384.

Hudson, J. A., and E. Liu, 1999, Effective elastic properties of heavily faulted structures: Geophysics, 64, 479-485.

Hudson, J. A., T. Pointer, and E. Liu, 2001, Effective-medium theories for fluid-saturated materials with aligned cracks: Geophysical Prospecting, 49, 509-522.

Kong, L., B. Gurevich, T. M. Müller, Y. Wang, H. Yang, 2013, Effect of fracture fill on seismic attenuation and dispersion in fractured porous rocks: Geophysical Journal International, 195, 1679-1688.

Krzikalla, F., and T. M. Müller, 2011, Anisotropic P-SV-wave dispersion and attenuation due to inter-layer flow in thinly layered porous rocks: Geophysics, 76, WA135-WA145.

Lambert, G., B. Gurevich, and M. Brajanovski, 2006, Attenuation and dispersion of P-waves in porous rocks with planar fractures: Comparison of theory and numerical simulations: Geophysics, 71, N41-N45.

Liu, E., and A. Martinez, 2013, Seismic fracture characterization: Concepts and practical applications: EAGE. 
Masson, Y. J., and S. R. Pride, 2007, Poroelastic finite difference modeling of seismic attenuation and dispersion due to mesoscopic-scale heterogeneity: Journal of Geophysical Research, 112.

Maultzsch, S., M. Chapman, E. Liu, and X. Y. Li, 2003, Modelling frequency-dependent seismic anisotropy in fluid-saturated rock with aligned fractures: implication of fracture size estimation from anisotropic measurements: Geophysical Prospecting. 51, 381-392.

Milani, M., L. Monachesi, J. I. Sabbione, J. G. Rubino, and K. Holliger, 2016, A generalized effective anisotropic poroelastic model for periodically layered media accounting for both Biot's global and interlayer flows: Geophysical Prospecting, 64, 1135-1148.

Müller, T. M., B. Gurevich, and M. Lebedev, 2010, Seismic wave attenuation and dispersion resulting from wave-induced flow in porous rocks: A review: Geophysics, 75, 147-164.

Nakagawa, S., and M. A. Schoenberg, 2007, Poroelastic modeling of seismic boundary conditions across a fracture: J. Acoust. Soc. Am. 122, 831-847.

Quintal, B., R. Jänicke, J. G. Rubino, H. Steeb, and K. Holliger, 2015, Sensitivity of Swave attenuation to the connectivity of fractures in fluid-saturated rocks: Geophysics, 79, WB15-WB24.

Rathore, J. S., E. Fjaer, R. M. Holt, and L. Renlie, 1995, P-and S-wave anisotropy of a synthetic sandstone with controlled crack geometry: Geophysical Prospecting, 43, 711-728.

Rubino, J. G., C. L. Ravazzoli, and J. E. Santos, 2009, Equivalent viscoelastic solids for heterogeneous fluid-saturated porous rocks: Geophysics, 74, N1-N13. 
Rubino, J. G., L. Guarracino, T. M. Müller, and K. Holliger, 2013, Do seismic waves sense fracture connectivity?: Geophysical Research Letters, 40, 692-696.

Rubino, J. G., G. A. Castromán, T. M. Müller, L. B. Monachesi, F. I. Zyserman, and K. Holliger, 2015, Including poroelastic effects in the linear slip theory: Geophysics, 80, A51-A56.

Rubino, J. G., E. Caspari, T. M. Müller, M. Milani, N. D. Barbosa, and K. Holliger, 2016, Numerical upscaling in 2-D heterogeneous poroelastic rocks: Anisotropic attenuation and dispersion of seismic waves: Journal of Geophysical Research: Solid Earth, 121, 66986721.

Schoenberg, M. A., 1980, Elastic wave behavior across linear slip interfaces: The Journal of the Acoustical Society of America, 68, 1516-1521.

Schoenberg, M., and J. Douma, 1988, Elastic wave propagation in media with parallel fractures and aligned cracks: Geophysical Prospecting, 36, 571-590.

Schoenberg, M., and C. M. Sayers, 1995, Seismic anisotropy of fractured rock: Geophysics, 60, 204-211.

Solazzi, S. G., J. G. Rubino, T. M. Müller, M. Milani, L. Guarracino and K. Holliger, 2016, An energy-based approach to estimate seismic attenuation due to wave-induced fluid flow in heterogeneous poroelastic media: Geophysical Journal International, 207, 823-832.

Thomsen, L., 1986, Weak elastic anisotropy: Geophysics, 51, 1954-1966.

Thomsen, L., 1995, Elastic anisotropy due to aligned cracks in porous rock: Geophysical Prospecting, 43, 805-829. 
Tillotson, P., M. Chapman, A. I. Best, J. Sothcott, C. McCann, W. Shangxu, and X. Y. Li, 2011, Observations of fluid-dependent shear-wave splitting in synthetic porous rocks with aligned penny-shaped fracture: Geophysical Prospecting, 59, 111-119.

Wenzlau, F., J. B. Altmann, and T. M. Müller, 2010, Anisotropic dispersion and attenuation due to wave-induced fluid flow: Quasi-static finite element modeling in poroelastic solids: Journal of Geophysical Research: Solid Earth, 115.

Worthington, M. H., and R. Lubbe, 2007, The scaling of fracture compliance: Geological Society (London): Special Publications, 270, 73-82.

White, J. E., N. Mihailova, and F. Lyakhovitsky, 1975, Low-frequency seismic waves in fluid-saturated layered rocks: The Journal of the Acoustical Society of America, 57, S30-S30. 